\title{
Echocardiography; Questions and Difficulties
}

\author{
João Carlos Hueb \\ Hospital das Clínicas da Faculdade de Medicina de Botucatu - UNESP, São Paulo, SP - Brazil
}

The first big mistake we have made over the years, while executing an echocardiography scan, has been determining ejection fraction using the cube formula by the M-mode. This formula admits that, as the left ventricle (LV) is an ellipsoid of revolution, its longitudinal diameter is twice the cross-section; therefore, by raising the transverse diameter of its base to the third power, we have exactly the volume of the chamber and, with the diameter (volume) in systole and diastole, the ejection fraction can be easily determined. ${ }^{1}$ Then, it was determined that that was not quite true: when the ventricle dilated, it lost the ellipsoid conformation and acquired a spherical shape making the cube formula completely inappropriate; that is, it only served to determine the ejection fraction in normal ventricles. Then, Teichholz appeared to save us and adjust the cube formula, developing a formula that allowed to determine the ejection fraction in the ventricles of any shape. ${ }^{2}$ It was then realized that if a patient had had a stroke with a huge apical aneurysm retaining most of the blood during systole, as long as the septal base and the posterior wall presented good contraction, the ejection fraction would be normal using the Teichholz formula. Once again, we were saved, evoking the 1757 formula of mathematician Simpson, used here to calculate the ventricular volume. The left ventricle is then sliced into two planes after the endocardial delimitation, considering all chamber segments. ${ }^{3}$ Although this method has virtually solved the ejection fraction calculation, it has limitations as the endocardium cannot always be viewed on echocardiogram. Still, it was thought that definitive solution had been found for the assessment of LV systolic function, until the speckle tracking appeared, showing that a patient may have systolic dysfunction of this chamber even with a normal ejection fraction. ${ }^{4}$

With echocardiography scans, the LV myocardial mass could also be easily determined through the brilliant formula of Devereux, ${ }^{5}$ which considers the septal and posterior wall thickness to calculate the diastolic diameter of the chamber. Patients with sigmoid septum (mainly older people) are often found. In these patients, the basal septal portion is thicker and, when that site is used to make the

\section{Keywords}

Echocardiography; Diagnosis; Stroke Volume; Ventricular Dysfunction.

Mailing Address: João Carlos Hueb •

Departamento de Clínica Médica - Campus da Unesp de Botucatu

Postal Code 18618-000, Distrito de Rubião Jr., Botucatu, SP - Brazil E-mail: jchueb@uol.com.br

Manuscript received February 15, 2016; revised March 9, 2016; accepted March 31, 2016. measurement, it is assumed that the rest of the wall is thick and the ventricular mass is the overestimated. It is not possible to obtain a correct plan to determine the ventricular diameter, conducting the measurement on oblique planes obtaining larger diameters than the actual one; the result is an overestimated myocardial mass. Maintaining the linear measurement methods with M-mode on the spotlight, the same failure is observed when measuring the left atrium, which may have a normal anteroposterior diameter and dimensions "consequently" normal and be dilated to the sides. This distortion is attenuated when atrial volume is considered. Even worse, people often think that everyone, regardless of their height and weight, have hearts in the same size, so that the normality parameters of the chamber diameters are the same for everyone.

How often did you come across with a mitral valve without any thickening on M-mode, therefore with no myxoid degeneration and you would relentlessly move the transducer until the $C D$ segment of the valve in M-mode acquired a downward concave shape, making prolapse? In the 1980s, nearly one-third of young women "had" this heart disease. Today, a reverse path is followed, undoing the prolapse diagnoses that were usually done in the past.

Now, the LV diastolic function analysis is actually hard to carry out. In the past, its diagnosis criteria was just the inversion of the E/A ratio of the mitral flow chart. As most people older than 60 presents this abnormality, a myriad of cardiac patients with LV diastolic dysfunction is fabricated. Fortunately, in 2009, the consensus of the American Society of Echocardiography, with the aid of tissue Doppler, put some things in order. ${ }^{6}$ Nevertheless, it is still a mess, with a large gray area interposed between normal and abnormal.

When it comes to quantifying valvar lesions, the difficulties remain. In determining the mitral valve area by pressure half-time, if there is an LV diastolic dysfunction, the mitral flowchart E-wave decline ramp flattens out, and the valve area is underestimated. With the aortic valve, the same problem occurs: if there is LV systolic dysfunction, a very serious stenosis with a not very high valve gradient may emerge. Everything is further complicated when aortic stenosis is combined with normal ejection fraction and systolic dysfunction on speckle tracking - a method that is not used routinely to evaluate this valve - making the gradient to underestimate the stenosis severity. Similarly, when there is right ventricle (RV) systolic dysfunction, the determination of the pressure in the pulmonary artery through the tricuspid reflux - which is actually the RV pressure - is compromised, not reflecting the degree of pulmonary circulation resistance.

In this case, one should not believe that echocardiography is a flawed method. On the contrary, it is a wonderful method

DOI: $10.5935 / 2318-8219.20160028$ 
that introduced innovations in cardiology, making the diagnosis of heart diseases much easier and accurate. However, it is increasingly necessary to realize that, much more important than numbers, is the subjective analysis that is done when performing this test. With it, one can accurately infer whether a systolic dysfunction is mild, moderate or severe, and whether ventricular hypertrophy or chamber dilatation, solely by visual analysis, without measuring anything. I wonder if anyone wouldn't successively measure ejection fraction by the Simpson method and only stopped measuring it to find a number that was according to their subjective impression? Unfortunately, the accuracy of delivering a diagnosis using visual analysis alone can only be acquired with time, and in such circumstances, intuition is actually the best aid.

\section{Authors' contributions}

Manuscript drafting: HUEB JC.

\section{Potential Conflicts of Interest}

There are no relevant conflicts of interest.

\section{Sources of Funding}

This study had no external funding sources.

\section{Academic Association}

This study is not associated with any graduate program.

\section{References}

1. Pombo JF, Troy BL, Russell RO Jr. Left ventricular volumes and ejection fraction by echocardiography. Circulation. 1971;43(4):480-90.

2. Teichholz LE, Kreulen T, Herman MV, Gorlin R: Problems in echocardiographic volume determinations: echocardiographic-angiographic correlations in the presence or absence of asynergy. Am J Cardiol 1976;37(1):7-11.

3. Wyatt HL, Heng MK, Meerbaum S, Gueret P, Hestenes J, Dula E, et al. Cross-sectional echocardiography. Analysis of mathematic models for quantifyng volume of the formalin-fixed left ventricle. Circulation. 1980;61(6):1119-25.
4. Kang Y, Cheng L, Li L, Chen H, Sun M, Wei Z, et al. Early detection of anthracycline-induced cardiotoxicity using two-dimensional speckle tracking echocardiography. Cardiol J. 2013;20(6):592-9.

5. Devereux RB, Alonso DR, Lutas EM, Gottlieb GJ, Campo E, Sachs I, et al. Echocardiographic assessment of left ventricular hypertrophy: comparison to necropsy findings. Am J Cardiol. 1986;57(6):450-8.

6. Nagueh SF, Appleton CP, Gillebert TC, Marino PN, Oh KK, Smiseth OA, et al. Recommendations for the evaluation of left ventricular diastolic function by echocardiography. J Am Soc Echocardiogr. 2009;22(2):107-33. 